\title{
MINIREVIEW
}

\section{CELLULOSE DIGESTION IN INSECTS}

\author{
Michael M. Martin \\ Division of Biological Sciences, University of Michigan, Ann $\Lambda$ rbor, \\ MI 48109, USA
}

(Received 20 Septenbor 1982)

\begin{abstract}
Cellulose digestion has been demonstrated in the Thysanura (Lepismatidae), Orthoptera (Cryptocercidac, Blattidac). Isoptera (Mastotermitidae, Kalotermitidae. Hodotermitidae, Rhinotermitdae. Termitidae), Coleoptera (Buprestidae. Anobiidae, Scarabaeidae, Cerambycidae), and Hymenoptera (Siricidae).

2. In all but the scarab beetles, cellulose digestion is brought about by a complex of three types of enzymes $\left(C_{1}\right.$-cellulases, $C_{r}$-cellulases, and cellobiases $)$ as in fungi.

3. Many insects are able to synthesize their own $C_{x}$-cellulases and cellobiases, but few (if any) can synthesize $C_{1}$-cellulases.

4. Insects compensate for their inability to synthesize $C_{1}$-cellulases by exploiting the cellulolytic potential of protozoa, bacteria, or fungi.

5. The maintenance of permanent populations of hindgut protozoa, the maintenance of permanent populations of hindgut bacteria, and the ingestion of fungal cellulases are described as three distinct mechanisms by which insects have been shown to use the cellulolytic potential of microorganisms.

6. A process in which ingested cellulolytic bacteria proliferate in one region of the gut at the expense of ingested cellulose, only to be digested and assimilated in a more posterior section, is a fourth possible mechanism by which insects might accomplish the digestion of cellulose with the help of microorganisms.
\end{abstract}

\section{INSECTS THAT DIGEST CELLULOSE}

The ability of many insects to thrive on wood, foliage and detritus has naturally stimulated investigations of the extent to which such species are able to digest the structural polysaccharides in their food. Species that have been shown to possess a capacity to digest cellulose are listed in Table 1. Based upon the values of the approximate digestibilities or assimilation efficiencies that have been determined for a few species, it appears that termites are more efficient cellulose digesters than the wood-boring beetles and siricid wood wasps. The silverfish, Ctenolepisma lineata, and the American cockroach, Periplaneta americana, are omnivorous species that readily include both cellulosic and non-cellulosic items in their diets. The rest of the species listed in Table 1 are wood-feeders. Foliage and detritus-feeders are conspicuously absent. No evidence for significant digestion of cellulose by foliage-feeding Orthoptera. Coleoptera or Lepidoptera or by detritus-feeding Plecoptera, Coleoptera, Diptera or Trichoptera has been reported, although low levels of hydrolytic activity toward cellulose powder have been detected in the gut fluids of a few species of locusts (Evans \& Payne, 1964: Morgan. 1975a, 1976). The grasshopper, Melanoplus bivittatus, is able to degrade hypocotl cell walls of bean seedlings, but there is no evidence to indicate that cellulose is among the cell wall constituents digested (Talmadge \& Albersheim, 1969). The absence of any enzymatic activity toward cellulose powder in the digestive fluids of a number of Lepidopteran larvae has been noted (Shinoda, 1930; Babers \& Woke, 1937; Mathur, 1966; Chattoraj \& Mall, 1969; Khan
\& Kasting, 1969; Burton et al., 1977; Dixit \& Mall, 1978; Mall et al., 1978). McGinnis \& Kasting (1969) conclusively demonstrated the inability of the pale western cutworm, Agrotis orthogonia, to digest cellulose by observing that less than $0.5 \%$ of the carbon- 14 from ingested labelled cellulose was respired in the form of ${ }^{14} \mathrm{CO}_{2}$. Although wood-feeders dominate the list of celluluse digesters, there are many species of insects which feed upon bark, phloem tissue, and wood that are unable to digest the cellulose they consume. Beetles from the families Bostrychidae, Curculionidae, Lyctidae and Scolytidae exemplify such species (Parkin, 1940; Chararas, 1979).

\section{THE CELLULOLYTIC ENZYMES OF INSECT GUT FLUIDS}

Cellulose digestion in insects is generally accomplished by a collection of enzymes believed to be similar to the ones responsible for cellulolytic ability in fungi (Table 2). The "cellulase complex" includes three major classes of hydrolytic enzymes: endoglucanases $\left(C_{x}\right.$-cellulases $)$ cellobiohydrolases ( $\mathrm{C}_{1}$-cellulases), and $\beta$-glucosidases (cellobiases) (Reese \& Mandels, 1971; Wood \& McCrae, 1979; Ghose et $a l,, 1981)$. The digestion of native cellulose is believed to be initiated when endoglucanases attack isolated amorphous regions of the predominantly crystalline cellulose matrix, creating nick sites in the linear cellulose chains. The cellobiohydrolases attack at the nick sites liberating cellobiose, exposing additional potential sites for attack by the endoglucanase, and generally disrupting the highly ordered structure of the 
cellulose aggregates. The continued combined action of the $C_{1}$ - and $C_{x}$-cellulases results in the eventual complete degradation of the original cellulose and the production of cellobiose and a mixture of soluble linear oligosaccharides of varying chain lengths. The cellobiose, which is a potential inhibitor of the $C_{1}$ " and $C_{x}$-cellulases, is hydrolyzed to glucose by cellobiase, and the various oligosaccharides are further degraded, ultimately to glucose, by the action of both the $C_{x}$-cellulases and the cellobiases. Thus, the utilization of cellulose is dependent upon the concerted and synergistic action of three types of enzymes. If any one of the three is missing. cellulose digestion cannot occur.

The presence of a complete cellulase complex in an insect's digestive juices is indicated by the capacity of the gut fluid or a cell-free extract of gut contents to liberate reducing sugars from, or to solubilize such forms of crystalline cellulose as, Avicell ${ }^{\circledR}$, filter paper or cotton. The presence of the entire cellulase complex is generally taken as evidence for the presence of enzymes comparable to the $C_{1}$-cellulases of fungi, that is enzymes specific for crystalline cellulose. It is now common practice to refer to such enzyme from insect gut fluids as $C_{1}$-cellulases, although to date none has actually been shown to be a cellobiohydrolase. Digestive $\mathrm{C}_{x}$-cellulases are conveniently detected in insects by assaying gut fluids for the capacity to degrade carboxymethylcellulose (CMC) or some form of swollen, amorphous, or reprecipitated cellulose. Cellobiase ativity is readily assayed by measuring the capacity of gut fluid to hydrolyze cellobiose to glucose.

When subjected to the appropriate assay pro- cedures, gut fluids from a number of cellulose digesting insects have been found to exhibit activity attributable to $C_{x}$-cellulases, $C_{1}$-cellulases, and cellobiases. One notable exception is the scarab beetle, Oryctes nasicornis, in which no soluble cellulases of any kind have been detected. In this insect, cellulose digestion is thought to be brought about by hindgut bacteria (Bayon \& Mathelin, 1980). The cellulase complex of bacteria consists of only two principal components, endoglucanases or $C_{x}$-cellulases and $\beta$-glucosidases or cellobiases. Cellobiohydrolases or $C_{1}$-cellulases have been demonstrated in only a few species of bacteria (Ghose et al., 1981). Furthermore, bacteria produce cell-bound as well as extracellular enzymes. The failure to detect any cellulases in the gut fluids of $O$. nasicornis could be explained if cellulose digestion is accomplished by the cell-bound enzymes of bacteria that are attached to the fragments of plant tissuc present in the insect's hindgut, and not by soluble enzymes presented in the gut fluids. This species provides an emphatic reminder that the failure to detect cellulolytic activity in an insect's digestive fluids is not compelling evidence against the digestion of dictary cellulose, especially in species with abundant populations of bacteria housed in enlarged segments of the gut.

\section{THE SECRETION OF THE ENZYMES OF THE CELLULASE COMPLEX BY INSECTS}

Table 3 is a comprehensive tabulation of insect species in which $C_{x}$-cellulase activity has been demonstrated in the gut fluids by assays using CMC or a

Table 1. Cellulose digesting insects

\begin{tabular}{lcc}
\hline $\begin{array}{c}\text { Order } \\
\text { Family } \\
\text { Species }\end{array}$ & $\begin{array}{c}\text { Evidence for } \\
\text { capacity to } \\
\text { digest cellulose }\end{array}$ & References \\
\hline
\end{tabular}

\begin{tabular}{|c|c|}
\hline THYSANURA & \\
\hline Lepismatidae & \\
\hline Ctenolepisma lineata & $\mathrm{AD}(72-87),{ }^{4} \mathrm{C}, \mathrm{EN}, i$ \\
\hline ORTHOPTERA & \\
\hline Cryptocercidae & \\
\hline Cryptoresers punctulatus & EN, NU \\
\hline Blattidae & \\
\hline Periplaneta americana & ${ }^{14} \mathrm{C}$ \\
\hline ISOPTERA & \\
\hline Mastotermitidae & \\
\hline Mastoternes darwiniensis & EN \\
\hline Kalotermitidae & \\
\hline Kalotermes flavicollis & $\mathrm{AD}(74-91)$ \\
\hline Neotermes bosei & EN \\
\hline Hodotermitidae & \\
\hline Zootermopsis angusticollis & $\operatorname{AD}(82), E N$ \\
\hline Rhinotermitidae & \\
\hline Coptotermes formosanus & ${ }^{14} \mathrm{C}$ \\
\hline Heferotermes indicolat & $\mathrm{AD}(78-89)$ \\
\hline Roticultermes flowipes & $\mathrm{AD}(91),{ }^{14} \mathrm{C}, \mathrm{EN}, \mathrm{NU}$ \\
\hline R. Iucifugus & $\mathrm{AD}(96-99), \mathrm{EN}, \mathrm{NU}$ \\
\hline R. sperutus & EN, NU \\
\hline Termitidac & \\
\hline Macrotermes natalensis & EN \\
\hline M. subhyalimus & EN \\
\hline Nastititermes ephratae & $\operatorname{AD}(91-97)$ \\
\hline Trinernitemes trinereaides & EN \\
\hline
\end{tabular}

Lasker \& Giese (1956)

Cleveland et al. (1934)

Bignell (1977)

Veivers et al. (1981)

Seifert \& Becker (1965)

Mishria (1979)

Trager (1932); Hungate (1938)

Mauldin et al. (1972)

Seifert \& Becker (1965)

Trager (1932); Esenther \& Kirk (1974);

Mauldin (1977)

Seifert \& Becker (1965); Orlova (1974)

Orlova (1974); Yamaoka \& Nagatani (1975)

Martin \& Martin $(1978,1979)$

Abo-Khatwa (1978)

Seifert \& Becker (1965)

Potts \& Hewitt $(1973,1974 a, b)$ 


\begin{tabular}{|c|c|c|}
\hline $\begin{array}{l}\text { Order } \\
\text { Family } \\
\quad \text { Species }\end{array}$ & $\begin{array}{l}\text { Evidence for } \\
\text { capacity to } \\
\text { digest cellulose }\end{array}$ & References \\
\hline \multicolumn{3}{|l|}{ COLEOPTERA } \\
\hline \multicolumn{3}{|l|}{ Buprestidae } \\
\hline Capnodis sp. & EN & Rivnay (1945) \\
\hline Chalcophora mariana & EN & Schlottke (1945) \\
\hline \multicolumn{3}{|l|}{ Anobiidae } \\
\hline Anobium punctatum & $\mathrm{AD}(33), \mathrm{EN}$ & Parkin (1940); Spiller (1951) \\
\hline A. striatum & $\mathrm{AD}(31)$ & Müller (1934) \\
\hline Ernobius mollis & $\mathrm{EN}$ & Parkin $(1940)$ \\
\hline Ptilinus pectiniromis & FN & Parkin $(1940)$ \\
\hline Xestobium rufovillosum & $\mathrm{AD}(49) . \mathrm{EN}$ & Norman (1936); Parkin (1940) \\
\hline \multicolumn{3}{|l|}{ Scarabaeidae } \\
\hline Oryctes nasicomis & $\operatorname{AD}(68),{ }^{14} \mathrm{C}$ & Rössler (1961); Bayon \& Mathelin (1980) \\
\hline Sericesthis geminata & EN & Soo Hoo \& Dudzinski (1967) \\
\hline \multicolumn{3}{|l|}{ Cerambycidae } \\
\hline Acanthocinus aedilis & EN & Schlottke (1945) \\
\hline Aegosoma scabriconae & EN & Ivanovic \& Barbic (1966) \\
\hline Cerambyx cerdo & EN & Ripper (1930); Müller (1934) \\
\hline Ergates faber & EN & Schlottke (1945); Chararas \& Libois (1976) \\
\hline Gracilia minuta & $\mathrm{AD}(33)$ & Müller (1934) \\
\hline Hylotrupes bajulus & $\mathrm{AD}(12-21)$ & Falck (1930): Becker (1942) \\
\hline Leptura rubra & $\mathrm{AD}(33)$ & Muiller (1934) \\
\hline Macrotoma palmata & $\mathrm{AD}(14-47)$ & Mansour \& Mansour-Bek (1934a) \\
\hline Morimus funerus & EN & Ivanovic \& Barbic (1966) \\
\hline Oxymirus cursor & $A D(49), E N$ & Müller (1934) \\
\hline Phymatodes testaceus & EN & Parkin $(1940)$ \\
\hline Plagionotus detritus & EN & Schlottke (1945); Ivanovic \& Barbic (1966) \\
\hline Rhagium bifasciatum & EN & Ripper (1930); Müller (1934) \\
\hline R. inquisitor & EN & Deschamps (1944): Schlottke (1945) \\
\hline R. nordax & EN & Parkin (1940); Schlottke (1945) \\
\hline Saperda populinae & EN & Schlottke (1945) \\
\hline Smodicum cucujiforme & $\mathrm{EN}$ & Parkin $(1940)$ \\
\hline Stromatium barbatum & $\mathrm{AD}(30-57) . \mathrm{EN}$ & Mishra \& Singh (1978) \\
\hline S. fulvum & EN & Mansour \& Mansour-Bek (1937) \\
\hline Xylotrechus rusticus & EN & Parkin $(1940)$ \\
\hline \multicolumn{3}{|l|}{ HYMENOPTERA } \\
\hline \multicolumn{3}{|l|}{ Siricidae } \\
\hline Sirex cyaneus & EN & Kukor \& Martin (1983) \\
\hline S. gigas & $\mathrm{AD}(22)$ & Müller (1934) \\
\hline S. phantoma & $\mathrm{AD}(31)$ & Müller (1934) \\
\hline
\end{tabular}

Abbreviations: AD, cellulose digestion demonstrated by comparing the cellulose contents of food and frass; the number in the parenthesis is the approximate digestibility of cellulose; ${ }^{14} \mathrm{C}$, cellulose digestion demonstrated by noting the production of ${ }^{14} \mathrm{CO}_{2}$ or the incorporation of ${ }^{14} \mathrm{C}$ into tissues following ingestion of $\mathrm{U}^{14} \mathrm{C}$-cellulose; $\mathrm{EN}$, gut fluid demonstrated to possess the enzymatic capacity to degrade filter paper, cotton, Avicell ${ }^{\circledR}$ or some other form of crystalline cellulose; NU, ability to digest cellulose inferred from capacity to survive on a diet of pure cellulose.

suitable form of amorphous cellulose as the test substrate. It is a long list that includes not only familiar cellulose-digesting species, such as termites, wood roaches and cerambycid beetles, but also many species from groups which are not thought to be capable of assimilating cellulose. In a number of the investigations summarized in Table $3, \mathrm{C}_{x}$-cellulase activity was found to be present in extracts of salivary glands and midgut tissues, indicating that the enzymes were produced by the insects and not by microbial symbionts residing in the gut. $\mathrm{C}_{\mathrm{x}}$-cellulases of insect origin have been demonstrated in 17 species of roaches, 8 termites. and 31 aphids. To be sure, in many of the species listed, $C_{x}$-cellulase activity is low may be due to enzymes which normally exert their catalytic action on non-cellulosic poly- or oligosaccharides. Nonetheless, it seems clear that the presence of enzymes with $C_{x}$-cellulase activity is not unusual in insect gut fluids, and that the rather restricted occurrence of the ability to digest cellulose in insects is not due to the restricted distribution of this class of enzymes.

Cellobiase activity has also been detected in the gut fluids of a diverse array of insect species, both digesters and non-digesters of cellulose, and has been demonstrated in extracts of salivary glands or midgut tissues from 2 species of locusts, 6 termites, one pyrrhocorid bug, the larvae of one sciarid fly, and even from silkworms (Table 4). $\beta$-Glucosidase activity has been detected in the gut fluids, gut tissues, and salivary glands of many additional species, and it is very probable that cellobiose would be hydrolyzed by the digestive fluids of many of these species as well. Thus, the presence of enzymes able to hydrolyze cellobiose are of common occurrence in insects. and the inability of most insects to digest cellulose cannot be attributed to the narrow distribution of the requisite cellobiases. 
Table 2. Enzymes of cellulose digestion in fungi and probably also in insects

Enzyme

(Alternate designations)

Mode of action and products

Substrates

The cellulase complex

1,4- $\beta$-D-Glucan 4-glucanohydrolase (F.C 3.2.1.4)

(Endo- $\beta$-1,4-Glucanase)

(Endoglucanase)

(Carboxymethylcellulase)

(CMCase)

$\left(C_{x}\right.$-Cellulase $)$

\author{
1.4- $\beta$-1D-Glucan cellobiohydrolase \\ (EC 3.2.1.91) \\ (Cellobiohydrolase) \\ ( $\mathrm{C}_{1}$-cellulase)
}
1.4- $\beta$-D-Glucoside 4-glucohydrolase (EC 3.2.1.21)
$(\beta-\mathrm{D}$-Glucosidase)
(Cellobiase)

A combination of the three categories brings about the complete digestion of native cellulose to glucose.

Random attack on $\beta$-1,4-glucosidic bonds, generating transient cellodextrins, cellobiose, and glucose.

Removal of cellobiose units from the non-reducing end of a linear chain by attack on penultimate $\beta$-1,4-glucosidic bonds. of enzymes designated below, which

\begin{abstract}
Microcrystalline cellulose powder, Avicell ${ }^{\circledR}$, cotton, and filter paper.
\end{abstract}

CMC and other soluble derivatives of cellulose. phosphoric acid swollen cellulose, and cellodextrins (increasing activity with increasing chain length). No activity toward crystalline cellulose. Hardly any activity toward cellobiose.

Microctystalline cellulose powder, Avicell ${ }^{\circledast}$, cotton, swollen cellulose, and cellodextrins

(increasing activity with increasing chain length). Limited activity toward CMC.

Hydrolysis of the $\beta-1,4-$ glucosidic bond of cellobiose to generate glucose.
Cellobiose, other $\beta$-linked disaccharides of glucose, and cellodextrins. No activity toward cellulose

Table 3. Insects with digestive $\mathrm{C}_{\mathrm{x}}$-cellulases

\begin{tabular}{cccc}
\hline $\begin{array}{c}\text { Insect Order } \\
\text { Family } \\
\text { Species }\end{array}$ & $\begin{array}{c}\text { Capacity } \\
\text { to digest } \\
\text { cellulose }\end{array}$ & $\begin{array}{c}\text { Identified sources } \\
\text { of } C_{x} \text {-cellulases in } \\
\text { digestive fluids }\end{array}$ & References \\
\hline
\end{tabular}

ORTHOPTERA

Crypocercus punctulatus

Blattidae

Blatta orientalis

Periplaneta americana

P. australasiae

P. fuliginosa

Blaberidae

Blaherus craniafer

B. discoidalis

B. giganticus

Byrostria funigata

Capucina patula

Diploptera punctata

Eublaberus posticus

Gromphadorhina branneri

Letacophaea maderae

Nauphota cinera

Phortioeca phoraspoides

Pycnoscelus surinamensis

Acrididae

Locusta migratoria

Melanoplus bivittatus

Schistocerca gregaria

ISOPTERA

Mastotermitidae

Mastotermes darwiniensis

Kalotermitidae

Neotemes bosei
High

Unknown

Moderate

Unknown

Unknown

Unknown

Unknown

Unknown

Unknown

Unknown

Unknown

Unknown

Unknown

Unknown

Unknown

Unknown

Unknown

Limited or none

Limited or none

Limited or none

SG, HGP

SG

SG, GB

SG

SG

SG

SG

SG

SG

SG

SG

SG, GB

SG

SG

SG

SG

SG

Unknown (WA)

Unknown (WA)

Unknown (WN)
Trager (1932); Wharton \& Wharton (1965)

Wharton \& Wharton (1965)

Wharton \& Wharton (1965); Cruden \& Markovetz (1979)

Wharton \& Wharton (1965)

Wharton \& Wharton (1965)

Wharton \& Wharton (1965)

Wharton \& Wharton (1965)

Wharton \& Wharton (1965)

Wharton \& Wharton (1965)

Wharton \& Wharton (1965)

Wharton \& Wharton (1965)

Wharton \& Wharton (1965); Cruden \& Markovetz (1979)

Wharton \& Wharton (1965)

Wharton \& Wharton (1965)

Wharton \& Wharton (1965)

Wharton \& Wharton (1965)

Wharton \& Wharton (1965)

Morgan (1976)

Talmadge \& Albersheim (1969)

Evans \& Payne (1964)

Presumably high SG, MGT, HGP Veivers et al. (1981)

High SG, MGT Mishra (1980) 
Table 3-continued

\begin{tabular}{|c|c|c|c|}
\hline $\begin{array}{l}\text { Insect Order } \\
\text { Family } \\
\text { Species }\end{array}$ & $\begin{array}{l}\text { Capacity } \\
\text { to digest } \\
\text { cellulose }\end{array}$ & $\begin{array}{l}\text { Identified sources } \\
\text { of } \mathrm{C}_{\mathrm{x}} \text {-cellulases in } \\
\text { digestive fluids }\end{array}$ & References \\
\hline $\begin{array}{l}\text { Hodotermitidae } \\
\text { Hodotermes mossambicus } \\
\text { Rhinotermitidae }\end{array}$ & High & MGT & Botha \& Hewitt (1979) \\
\hline Coptotermes lacteus & Presumably high & MGT, HGP & O'Brien et al. (1979) \\
\hline Reticulitermes speratus & High & SG, HGP & Yokoe (1964); Yamaoka \& Nagatani (1975) \\
\hline R. hesperus & Presumably high & HGB & Thayer $(1978)$ \\
\hline Zootermopsis sp. & High & HGP & Yamin \& Trager (1979) \\
\hline \multicolumn{4}{|l|}{ Termitidae } \\
\hline Macrotermes natalensis & Presumably high & SG, MGT, IFT & Martin \& Martin $(1978,1979)$ \\
\hline M. subhyalinus & Presumably high & MGT, IFT & Abo-Khatwa (1978) \\
\hline Microcerotermes edentatus & Presumably high & Unknown & Kovoor $(1970)$ \\
\hline Nasutitermes exitiosus & High & MGT & O'Brien et al. (1979) \\
\hline Termes obesus & Presumably high & Unknown & Misra \& Ranganathan (1954) \\
\hline Trinevitermes trinervoides & Presumably high & MGT & Potts \& Hewitt $(1973,1974 a, b)$ \\
\hline \multicolumn{4}{|l|}{ PLECOPTERA } \\
\hline \multicolumn{4}{|l|}{ Pteronarcyidae } \\
\hline Allonarcys proteus & Limited or none & Unknown (WA) & Sinsabaugh et al. (1981) \\
\hline Pteronarcys californica & Limited or none & Unknown (WA) & Martin et al. (1981b) \\
\hline P. pictetei & Limited or none & Unknown (WA) & Martin et al. (1981b) \\
\hline \multicolumn{4}{|l|}{ PSOCOPTERA } \\
\hline \multicolumn{4}{|l|}{ Pseudocaeciliidae } \\
\hline \multicolumn{4}{|l|}{ HEMIPTERA } \\
\hline \multicolumn{4}{|l|}{ Pentatomidae } \\
\hline Palomena angulosa & Unknown & Unknown & Hori (1975) \\
\hline Eurydema rugosum & Unknown & Unknown & Hori $(1975)$ \\
\hline \multicolumn{4}{|l|}{ Coreidae } \\
\hline Coreus marginatus & Unknown & Unknown & Hori $(1975)$ \\
\hline \multicolumn{4}{|l|}{ HOMOPTERA } \\
\hline \multicolumn{4}{|l|}{ Aphididae } \\
\hline Acyrthosiphon caragenae & Unknown & SG & Adams \& Drew (1965) \\
\hline A. pisum & Unknown & SG & Adams \& Drew (1965) \\
\hline Aphis fabae & Unknown & SG & Adams \& Drew (1965) \\
\hline A. helianthi & Unknown & SG & Adams \& Drew (1965) \\
\hline A. pomi & Unknown & SG & Adams \& Drew (1965) \\
\hline Aulacorthum solani & Unknown & SG & Adams \& Drew (1965) \\
\hline Betulaphis quadrituberculata & Unknown & SG & Adams \& Drew (1965) \\
\hline Calaphis (?) betulaecolens & Unknown & SG & Adams \& Drew (1965) \\
\hline Ductynotus cirsii & Unknown & SG & Adams \& Drew (1965) \\
\hline D. russellae & Unknown & SG & Adams \& Drew (1965) \\
\hline D. tararaci & Unknown & SG & Adams \& Drew (1965) \\
\hline$D, s p$ & Unknown & SG & Adams \& Drew (1965) \\
\hline Eriosoma lanigerum & Unknown & SG & Adams \& Drew (1965) \\
\hline Hydaphis foenicul & Unknown & SG & Adams \& Drew (1965) \\
\hline Macrosiphon calfornicum & Unknown & SG & Adams \& Drew (1965) \\
\hline M. euphorbia & Unknown & SG & Adams \& Drew (1965) \\
\hline M. ptericoleus & Unknown & SG & Adams \& Drew (1965) \\
\hline Metopolophium dirhodum & Unknown & SG & Adams \& Drew (1965) \\
\hline Myzocallis walshii & Unknown & SG & Adams \& Drew (1965) \\
\hline Mysus cerasi & Unknown & SG & Adams \& Drew (1965) \\
\hline$M$. persicae & Unknown & SG & Adams \& Drew (1965) \\
\hline Nearctaphis bakeri & Unknown & SG & Adams \& Drew (1965) \\
\hline Neomyzus circumflexus & Unknown & SG & Adams \& Drew (1965) \\
\hline Pentatrichopus thomasi & Unknown & SG & Adams \& Drew (1965) \\
\hline Periphylus lyropictus & Unknown & $\mathrm{SG}$ & Adams \& Drew (1965) \\
\hline P. negundinus & Unknown & SG & Adams \& Drew (1965) \\
\hline Prociphilus leselata & Unknown & SG & Adams \& Drew (1965) \\
\hline Pferocomma bicolor & Unknown & SG & Adams \& Drew (1965) \\
\hline Rhopalosiphum sp. & Unknown & SG & Adams \& Drew (1965) \\
\hline R. padi & Unknown & SG & Adams \& Drew (1965) \\
\hline \multirow{2}{*}{\multicolumn{4}{|c|}{ COLEOPTERA }} \\
\hline & & & \\
\hline \multicolumn{4}{|l|}{ Scarabaeidae } \\
\hline Sericesthis geminata & Unknown & Unknown & Soo Hoo \& Dudzinski (1967) \\
\hline \multicolumn{4}{|l|}{ Cerambycidae } \\
\hline Ergates faber & $\begin{array}{l}\text { Presumably } \\
\text { moderate }\end{array}$ & Unknown & Chararas (1979) \\
\hline
\end{tabular}


Table 3-continued

\begin{tabular}{|c|c|c|c|}
\hline $\begin{array}{l}\text { Insect Order } \\
\text { Family } \\
\text { Species }\end{array}$ & $\begin{array}{l}\text { Capacity } \\
\text { to digest } \\
\text { cellulose }\end{array}$ & $\begin{array}{l}\text { Identified sources } \\
\text { of } C_{x} \text {-cellulases in } \\
\text { digestive fluids }\end{array}$ & References \\
\hline \multirow[t]{2}{*}{ Phoracantha semipunctata } & \multirow{2}{*}{$\begin{array}{l}\text { Presumably } \\
\text { moderate } \\
\text { Presumably } \\
\text { moderate }\end{array}$} & Unknown & Chararas (1979) \\
\hline & & Unknown & Chararas (1979) \\
\hline Stromattum barbatum & Moderate & Unknown & Mishra \& Singh (1978) \\
\hline \multicolumn{4}{|l|}{ Curculionidae } \\
\hline Cryptorrhyolus lapathi & Limited or none & Unknown & Chararas (1979) \\
\hline Hylohius abietes & Limited or none & Unknown & Chararas (1979) \\
\hline Pissodes hareynias & Limited or none & Unknown & Chararas (1979) \\
\hline P. notatws & Limited or none & Unknown & Chararas (1979) \\
\hline \multicolumn{4}{|l|}{ Scolytidac } \\
\hline Carphobonts minimus & Limited or none & Unknown & Churaras (1979) \\
\hline Ips umitinus & Limited or none & Unknown & Chararas (1979) \\
\hline 1. sexdentatus & Limited or none & Unknown & Chararas (1979) \\
\hline 1. typographus & Limited or none & Unknown & Chararas $(1979)$ \\
\hline Phlocosinus cedri & Limited or none & Unknown & Chararas (1979) \\
\hline Scolytus intricatus & Limited or none & Unknown & Chararas $(1979)$ \\
\hline S. multistriatus & Limited or none & Unknown & Chararas (1979) \\
\hline S. numblicus & Limited or none & Unknown & Chararas $(1979)$ \\
\hline S. solytus & Limited or none & Unknown & Charitras $(1979)$ \\
\hline \multicolumn{4}{|l|}{ MEGALOPTERA } \\
\hline \multicolumn{4}{|l|}{ Sialidie } \\
\hline Sialis hutaria & Limited or none & Unknown & Monk (1976) \\
\hline \multicolumn{4}{|l|}{ TRICHOPTERA } \\
\hline \multicolumn{4}{|l|}{ Limnephilidae } \\
\hline Extipsopteryx qutulata & Limited or none & Unknown (WA) & Bjarnov (1972) \\
\hline Halestes sp. & Limited or none & Unknown & Monk (1976) \\
\hline Potonophytax sp. & Limited or none & Unknown & Monk (1976) \\
\hline Sito nigrigornis & Limited or none & Unknown (WA) & Bjarnov $(1972)$ \\
\hline \multicolumn{4}{|l|}{ Rhyacophilidae } \\
\hline Rhyouphila septuntronis & Limited or none & Unknown (WA) & Bjarnov (1972) \\
\hline \multicolumn{4}{|l|}{ Brachycentridac } \\
\hline \multicolumn{4}{|l|}{ Polycentropodidae } \\
\hline Noure fipsis angustipennis & Limited or none & Unknown & Bjamov (1972) \\
\hline Plectrochemia genciculata & Limited or none & Unknown & Monk (1976) \\
\hline Polvcentropus ftoromaculatus & Limited or none & Unknown & Monk (1976) \\
\hline \multicolumn{4}{|l|}{ Hydropsychidae } \\
\hline Hydropsuche anyustipenais & Limited or none & Unknown & Bjarnov $(1972)$ \\
\hline \multicolumn{4}{|l|}{ Phryganeidae } \\
\hline Atylpuia vestitu & Limited or none & Unknown & Martin et al. (1981a) \\
\hline Phryanea sp. & Limited or none & Unknown & Martin et al. (1981a) \\
\hline \multicolumn{4}{|l|}{ DIPTERA } \\
\hline \multicolumn{4}{|l|}{ Tipulidae } \\
\hline Tipula abdommatis & Limited or none & Unknown (WA) & Sinsabaugh ef al. (1981) \\
\hline $\begin{array}{l}\text { Chironomidae } \\
\text { Chinomomis anthrocinus }\end{array}$ & & & \\
\hline $\begin{array}{l}\text { Chimomoms anthrocinus } \\
\text { Sciaridae }\end{array}$ & Limited or none & Unknown & Bjarnov $(1972)$ \\
\hline $\begin{array}{l}\text { Rhymosciara americama } \\
\text { HYMFNOPTFRA }\end{array}$ & Limited or none & Unknown & Terra et al. (1979) \\
\hline Siricidate & & & \\
\hline Sirex cyunets & Moderate & IFT & Kukor \& Martin (1983) \\
\hline Eurytomidae & & & \\
\hline Eutytoma amyodali & Unknown & Unknown & Ishatad \& Plant (1974) \\
\hline
\end{tabular}

Abbreviations: WA, weak activity; GB, gut bacteria; HGB, hindgut bacteria: HGP. hindgut protozoa; IFT, ingested fungal tissue; MGT, midgut tissue; SG, salivary glands.

As indicated in Table 1 , the gut fluids of a number of insects, including the silverfish, the wood roach, several termites. and quite a few beetles, are able to effect the degradation of microcrystatline cellulose. Therefore, $C_{1}$-cellulases or comparable enzymes must be present. In contrast to the numerous reports of $C_{x}$ cellulase and cellobiose production by insects. data suggesting that insects are able to secrete their own $C_{1}$-cellulases exist for only three species, and even in these the evidence is not completely unam- 
Table 4. Insects with digestive cellobiases

\begin{tabular}{|c|c|c|c|}
\hline $\begin{array}{l}\text { Insect } \\
\text { Order } \\
\text { Family }\end{array}$ & $\begin{array}{l}\text { Capacity } \\
\text { to digest } \\
\text { cellulose }\end{array}$ & $\begin{array}{l}\text { Identified sources } \\
\text { of cellobiases in } \\
\text { digestive fluids }\end{array}$ & References \\
\hline \multicolumn{4}{|l|}{ THYSANURA } \\
\hline $\begin{array}{l}\text { Ctenolepisma lineata } \\
\text { ORTHOPTERA }\end{array}$ & Moderate & Unknown & Lasker \& Giese (1956) \\
\hline \multicolumn{4}{|l|}{ Blaberidae } \\
\hline $\begin{array}{l}\text { Byrostria fimigata } \\
\text { Acrididae }\end{array}$ & Acrididae & Unknown & Fisk \& Rato (1964) \\
\hline Locusta migratoria & Limited or none & MGT & Morgan (1975b) \\
\hline \multicolumn{3}{|l|}{ ISOPTERA } & Evans \& Payne (1964) \\
\hline \multicolumn{4}{|l|}{ Mastotermitidae } \\
\hline \multicolumn{3}{|l|}{ Kalotermitidae } & Veivers et al. (1981) \\
\hline $\begin{array}{l}\text { Neotermes bosei } \\
\text { Rhinotermitidae }\end{array}$ & High & MGT & Mishra (1980) \\
\hline $\begin{array}{l}\text { Coptotermes lacteus } \\
\text { Termitidae }\end{array}$ & Presumably high & HGP & McEwen et al. (1980) \\
\hline Mutroterntes subhyalinus & Presumably high & MGT & Abo-Khatwa (1978) \\
\hline Microcerotemes edentatus & Presumably high & Unknown & Kovoor $(1970)$ \\
\hline Nasutiternes exitiosus & High & Probably MGT & McEwen et al. (1980) \\
\hline N. walkeri & Presumably high & Probably MGT & McEwen et al. $(1980)$ \\
\hline $\begin{array}{l}\text { Trinervitermes trinervoides } \\
\text { PSOCOPTERA }\end{array}$ & \multicolumn{2}{|c|}{ PSOCOPTERA } & Potts \& Hewitt (1973) \\
\hline \multicolumn{4}{|l|}{ Pseudocaeciliidae } \\
\hline \multicolumn{4}{|l|}{ HEMIPTERA } \\
\hline \multicolumn{4}{|l|}{ Miridate } \\
\hline \multicolumn{4}{|l|}{ Pyrrhocoridae } \\
\hline Disdercus fasciatus & Unknown & SG & Khan \& Ford (1967) \\
\hline \multicolumn{4}{|l|}{ COLEOPTERA } \\
\hline Brosus cephalotes & Unknown & Unknown & Nielsen (1962) \\
\hline Pterostichus oblongopunctatus & Unknown & Unknown & Nielsen (196?) \\
\hline \multicolumn{4}{|l|}{ Staphylinidae } \\
\hline \multicolumn{4}{|l|}{ Scarabaeidae } \\
\hline Sericesthis geminato & Unknown & Unknown & Soo Hoo \& Dudzinski (1967) \\
\hline \multicolumn{4}{|l|}{ TRICHOPTERA } \\
\hline Crunoecia irrorata & Limited or none & Unknown & Bjarnov (1972) \\
\hline Ecclipsopteryx quttaluta & Limited or none & Unknown & Bjarnov (1972) \\
\hline Chaetopteryx cillosa & Limited or none & Unknown & Bjarnov (1972) \\
\hline Potamophylax nigricornis & Limited or none & Unknown & Bjarbov (1972) \\
\hline Silo nigricomis & Limited or none & Unknown & Bjarnov (1972) \\
\hline \multicolumn{4}{|l|}{ Rhyacophilidae } \\
\hline \multicolumn{4}{|l|}{ Brachyceniridae } \\
\hline $\begin{array}{l}\text { Oligoplectum maculatum } \\
\text { Polycentripodidae }\end{array}$ & Limited or none & Unknown & Bjarnov $(1972)$ \\
\hline $\begin{array}{l}\text { Neureclepsis bimaculata } \\
\text { Hydropsychidae }\end{array}$ & Limited or none & Unknown & Bjarnov $(1972)$ \\
\hline Hydropsyche angustipennis & Limited or none & Unknown & Bjarnov (1972) \\
\hline $\begin{array}{l}\text { LEPIDOPTERA } \\
\text { Bombycidae }\end{array}$ & & & \\
\hline $\begin{array}{l}\text { Bombyx mori } \\
\text { DIPTERA }\end{array}$ & Limited or none & $\mathrm{SG}$ & Mukajyama (1961) \\
\hline Chironomidae & & & \\
\hline Chironomus anthracinius & Limited or none & Unknown & Bjarnov (1972) \\
\hline C. plumosus & Limited or none & Unknown & Bjarnov (1972) \\
\hline Sciaridae & & & \\
\hline $\begin{array}{l}\text { Rhyncosciara americana } \\
\text { HYMENOPTERA }\end{array}$ & Limited or none & MGT & Ferreira \& Terra (1980) \\
\hline Tenthredinidae & & & \\
\hline Diprium pini & Unknown & Unknown & Schulze \& Ehrhardt (1963) \\
\hline
\end{tabular}

Abbreviations: HGP, hindgut protozoa; MGT, midgut tissue; $\mathrm{SG}$, salivary glands. 
biguous and compelling. The silverfish, Ctenolepisma lineata, is the best candidate for a genuine $C_{1}$-cellulase producer. Silverfish have simple guts with no enlarged segments or blind sacs which might function as fermentation chambers. Lasker \& Giese (1956) were unable to culture any cellulolytic bacteria from these insects, and were also able to demonstrate efficient cellulose digestion in nymphs which were presumed to be symbiont free, having been reared from surface sterilized eggs on a diet of sterile, dried rolled oats. Lasker and Giese were unable to culture any bacteria from the guts of their presumed symbiontfree nymphs, but they did not perform total direct counts of bacteria in the gut. 'I hus the possibility is not absolutely ruled out that the presumed symbiontfree specimens still contained cellulolytic microbes of a type which could not be isolated as viable colonies using the culturing methods employed. Also, the diet of sterile. rolled oats was assumed to be free of cellulolytic microbes and enzymes, although that assumption appears not to have been tested experimentally. Thus, while the case for $\mathrm{C}_{1}$-cellulase secretion by the midgut of Ctenolepisma lineata is quite convincing, it falls just short of being absolutely air-tight.

Potts \& Hewitt $(1973,1974 a, b)$ have suggested that the midgut of the higher termite, Trinervitermes trinervoides, secretes a single enzyme with both $C_{x}$ - and $\mathrm{C}_{1}$-cellulase activity. This proposal rests upon the claim that the $C_{1}$ - and $C_{x}$-cellulolytic activity present in a chromatographic fraction obtained during the purification of a homogenate of worker abdomens was due to a single enzyme, the same $C_{x}$-enzyme that hald previously been detected in an extract of midgut tissue.

Veivers et al. (1981) detected low levels of $C_{1}$-cellulase activity in an extract of the salivary glands of the primitive termite, Mastotermes darwiniensis. Only $10 \%$ of the total $C_{1}$-activity assayed in this termite was present in the salivary extract, however. Most of the activity $(73 \%)$ was in the hindgut, which houses an abundant protozoan population. The proposal that the $C_{1}$-cellulase activity in the salivary extract is due to enzymes secreted by the termites rests upon the assumption that no contamination of the salivary gland preparations occurred during dissection.

It is often stated that the wood-boring anobiid and cerambycid beetles secrete all of their own cellulases. While there is no evidence to preclude this possibility, neither is there any to support it. The assumption is based entircly upon the lack of any correlation between a capacity to digest cellulose and the presence of intracellular symbionts (Mansour \& Mansour-Bek. 1934b; Parkin, 1940). The origins of the $C_{1}$-cellulases of these insects remain completely unknown at the present time.

In summary then, while additional research may confirm the ability of a few insects to produce $\mathrm{C}_{1}$-cellulases, this ability is certainly not widespread, and should be regarded as the exception rather than the rule. Thus it seems evident that it is the inability to synthesize and secrete $C_{1}$-cellulases that explains the inability of most insects to digest cellulose. With the possible exception of the silverfish, insects which are able to assimilate cellulose do so by exploiting the ccllulolytic potential of protozoa and fungi which produce soluble $\mathrm{C}_{1}$-cellulases or of bacteria which digest cellulose without the necessary secretion of such enzymes.

\section{CONTRIBLTIONS OF PROTOZOA, BACTERIA AND FLNGI TO CELLLLOSE DIGESTION IN INSECTS}

Three distinctly different types of insect-microbial interactions have been shown to serve as mechanisms by which insects digest cellulose using the cellulolytic potential of microorganisms. Termites and wood roaches maintain permanent populations of cellulolytic protozoa in their hindguts. Scarab beetles and the American cockroach house permanent populations of bacteria in their hindguts. presumably including cellulolytic strains. The fungus-growing termites and the siricid wood wasps culture cellulolytic fungi and ingest cellulolytic enzymes when they consume their symbiont along with their food. A fourth mechanism which has not yet been demonstrated in insects, but which may prove to be important in certain species, is the rapid proliferation in the gut of cellulolytic bacteria ingested along with a cellulosic substrate, followed by the digestion of the bacteria in a more posterior section of the alimentary tract. Endosymbiotic bacteria or yeasts, housed in specialized cells (mycetocytes) or organs (mycetomes). represents a fifth type in insect-microbial interaction which is quite widespread. It was once thought that such symbionts might also be involved in cellulose digestion, but no evidence in support of that idea has ever been presented. The significance of such intracellular symbionts lies instead in their provision of B-vitamins, sterols. and essential amino acids.

The obligatory dependence of the lower termites and of the wood roach upon hindgut protozoa for cellulose digestion has been widely recognized since the classic investigations of Cleveland (1924). Cleveland et al. (1934). Trager (1932) and Hungate (1938. 1943), and has been thoroughly reviewed (Honigberg. 1970; O'Brien \& Slaytor 1982: Breznak. 1982). The protozoan symbionts are anaerobic species from unique genera of oxymonad. trichomonad and hypermastigote flagellates restricted to the four families of lower termites and to the wood roach family. Trager (1932) demonstrated that cellulolytic enzymes, including $\mathrm{C}_{1}$-cellulases. were produced by intestinal flagellates present in the roach. $C$. punctulatus, and in two species of termites, $R$. flawipes and $T$. anyusticollis. For many years it was not certain whether the enzymes were actually produced by the protozoa or by bacteria invariably present in the protoplasm of the protozoa. That uncertainty has recently been resolved by the successful cultivation of bacteria-free protozoa (Yamin \& Trager. 1979), and the clear demonstration that cellylolytic enzymes, including $C_{1}$-cellulases, are produced by the protozoa themselves and not by intracellular bacterial symbionts. Considering the impressive efficiency with which the exploitation of protozoan $C_{1}$-cellulases has allowed the lower termites to digest and assimilate cellulose, it seems surprising that this mechanism for acquiring cellulolytic capacity is not more widespread among wood-feeders. Perhaps a better understanding of the biochemical requirements of these unique protozoa, 
which should be possible to attain now that successful culturing methods have been developed, will explain the narrow phylogenetic distribution of this type of insect-microbial symbiosis.

It has often been presumed that cellulose digestion by the higher termites is accomplished by hindgut bacteria, since the Termitidae lack the xylophagous protozoa typical of the lower termites. However, evidence in support of this presumption is meager. Bacterial isolates with cellulolytic activity have been obtaned from a few termite species, but there are no data to suggest that such bacteria are of any quantitative importance to cellulose digestion in vivo (Lee \& Wood, 1971; Breznak, 1975, 1982; O'Brien \& Slaytor, 1982). Cellulolysis by resident hindgut bacteria has been proposed in the rhinocerus beetle, $O$. nasicomis (Bayon, 1980; Bayon \& Mathelin, 1980), and may occur in other scarabs as well (Wiedmann, 1930; Couturier, 1961), although cellulolytic bacteria have not actually been isolated from the intestinal tracts of these species. Gut bacteria, both from the midgut and hindgut, have been implicated in cellulose digestion in two species of roaches, $P$. americana (Bignell, 1977; Cruden \& Markovetz, 1979) and E. posticus (Cruden \& Markovetz. 1979). Many insects harbour abundant gut floras, and it is quite possible that further research will identify other species which exploit the cellulolytic capacities of their gut bacteria. However, it should be borne in mind that hindgut bacteria have also been shown to ferment sugars, fix nitrogen, degrade uric acid, synthesize amino acids, and participate in a host of biochemical processes besides cellulose digestion. Thus, the mere presence of an abundant gut flora from which cellulolytic strains of bacteria can be isolated does not constitute sufficient evidence to conclude that an insect derives any benefit from the cellulolytic potential of the bacteria it harbors within its alimentary tract.

Recently it has been demonstrated that the fungusgrowing termites Macrotermes natalensis (Martin \& Martin, 1978, 1979) and M. subhyalinus (Aba-Khatwa, 1978), and the larvae of the siricid wood wasp, Sirex cyaneus (Kukor \& Martin, 1983) acquire a capacity for cellulose digestion by ingesting fungal enzymes. The termites are able to produce some of their own cellobiases and $C_{x}$-cellulases, but the $C_{1}$-cellulases and some of the $C_{x}$-cellulases in their midgut fluids are derived from the conidiophores of a symbiotic fungus, Termitomyces sp., which the termites culture in their nests and consume in small quantities along with the wood and other cellulosic substrates which make up the bulk of their food. In like manner, wood wasps are associated with a symbiotic fungus which is the source of digestive $C_{1}-$ and $\mathrm{C}_{\mathrm{x}}$-cellulases, as well as hemicellulases and probably also pectinases, which allow the larvae to digest plant cell wall polysaccharides (Kukor \& Martin, 1983). The fungal symbiont of $S$. cyanews is Amylostereum chailletii. It is introduced into timber along with the wood wasp's egg during oviposition, and grows on the surfaces of the galleries produced by the feeding larvae. The larvae consume a mixture of wood and fungal mycelium. It is not known at present whether the ingestion of fungal cellulases is a common mechanism by which insects acquire a capacity to digest cellulose, or whether it is a process restricted to species involved in complex, highly coevolved symbiotic associations with fungi. It may prove to be very common (Martin, in press). Indeed, it is possible that many of the cerambycid beetles, which have been assumed to produce the entire complex of cellulases present in their digestive juices, acquire the $\mathrm{C}_{1}$-cellulases in their midgut fluids by ingesting fungal associates. Cellulases of fungal origin have been reported in the amphipod, Gammarus fossortm, an aquatic detritus-feeder (Bärlocher, 1982).

The proliferation in the gut of ingested bacteria has been demonstrated in the rhinocerus beetle, Oryctes nasicomis (Bayon \& Mathelin, 1980), and in several soil-feeding invertebrates, including the termite, Procubitermes aburiensis (Bignell et al., 1980), the millipede, Glomeris marginata (Anderson \& Bignell, 1980). and the isopod Tracheoniscus rathkei (Reyes \& Tiedje, 1976a). This proliferation of bacteria is accompanied by the degradation of hemicellulose and the assimilation of microbial cells in Tracheoniscus (Reyes \& Tiedje, 1976b). Thus, the indigestible constituents of the ingested detritus are transformed into microbial biomass which is digestible by the isopod. Although to date there has been no demonstration that the proliferation of ingested bacteria occurs at the expense of ingested cellulose, that possibility clearly exists, and needs to be considered as an additional mechanism by which insects might accomplish the digestion of cellulose by exploiting the metabolic capabilities of microorganisms. It is even possible that this process is responsible for the digestion of a portion of the ingested cellulose in the midgut of the rhinocerus beetle.

\section{CONCLUSION}

The investigation of insect-microbial interactions, pioneered with such elegance and insight by Buchner and Cleveland over a half a century ago, is experiencing something of a renaissance at the present time. Recent studies continue to uncover an ever growing number of ways by which insects exploit the biochemical characteristics of microorganisms, including their capacity to digest cellulose. These studies not only contribute to a greater understanding of the nutritional ecology of insects, but also promise to provide basic insights into the biochemical processes which mediate interactions between insects and microorganisms, and perhaps to help identify some of the factors which determine whether an interspecific interaction evolves into one of mutualism, commensalism or parasitism.

\section{REFERENCES}

Abo-Khatwa N, (1978) Cellulase of fungus growing termites: a new hypothesis on its origin. Experientia 34, $559-560$.

Adams J. B. \& Drew M. E. (1965) A cellulose-hydrolyzing factor in aphid saliva. Can. J. Tool 43, 489-496

ANDERSON J. M. \& BiGNell D. E. (1980) Bacteria in the food, gut contents and faeces of the litter-feeding millipede Glomeris marginata (Villers). Soil Biol. Biochem. 12 , $251-254$

Babers F. H. \& WOKE P. A. (1937) Digestive enzymes in the southern armyworm. J. agric. Res. 54, 547-550. 
BärLOCHer F. (1982) The contribution of fungal enzymes to the digestion of leaves by Gammans fossarum Koch. Oecologia 52, $1-4$

BAYON C. (1980) Volatile fatty acids and methane production in relation to anaerobic carbohydrate fermentation in Oryctes nasicomis larvae (Coleoptera: Scarabaeidae). J. Insect Physiol. 26, 819-828.

BAYON: C. \& MATHELin J. (1980) Carbohydrate fermentation and by-product absorption studied with labelled cellulose in Oryctes nasicormis larvae (Coleoptera: Scarabaeidael. J. Insect. Physiol. 26, 833-840.

BECKER G. (1942) Untersuchungen über die Ernahrungsphysiologie der Häusbockkafer, Z. vergl. Physiol. 29, 315-388.

BiGNeLL D. E. (1977) An experimental study of cellulose and bemicellulose degradation in the alimentary canal of the American cockroach. Can. J. Zool 55, 579-589.

Bignell D. L., Oskarsson H. \& Andersoy J. M. (1980) Distribution and abundance of bacteria in the gut of a soil-feeding termite Procubitermes aburiensis (Termitidae, Termitinae). J. gen. Microbiol. 117, 393-403.

Bjarnov N. (1972) Carbohydrases in Chironomus, Gammarus and some Trichopteran larvae. Oikos 23, 261-263.

Botha T. C. \& Hewitt P. H. (1979) Study of the gut morphology and some physiological observations on the influence of a diet of green Themeda triandra on the harvester termite, Hodotemes mossambicus (Hagen). Phyrophylactica 11, 57-60.

BREZNAK J. A. (1975) Symbiotic relationships between termites and their intestinal microbiota. Symp. Soc exp. Biol. 29, 559-580.

BREZNAK J. A. (1982) Intestinal microbiota of termites and other xylophagous insects. A. Rev. Microbiol. 36, $323-343$.

Burton R. L., Starks K. J. \& Sauer J. R. (1977) Carbohydrate digestion by the larval midgut of Heliothis zea. Ann. eni. Soc. Am. 70, 477-480.

Chararas (. (1979) Ecophysiologie des Insectes Parasites des Forêts. Published by the author. Paris.

Chararas C. \& Libois G. (1976) Studies on enzymes hydrolyzing glycosides in the larvae of Ergutes faber (Coleoptera: Cerambycidae). C. R. hebd. Séanc. Ac'ad. Sci., Paris 283, 1523-1525.

Chattorai A. N. \& MaII. S B. (1969) Hydrogen ion concentration and digestive enzymes in the mature larvae of Marasmia trapezalis Guen (Pyralidae). Indian J. Ent. 31, $121-126$.

Cleveland L. R. (1924) The physiological and symbiotic relationship between the intestinal protozoa of termites and their hosts, with special reference to Reticulitermes favipes Kollar, Biol. Bull, mar. hiol. Lab. Woods Hole 46, $117-227$.

Cleveland L. R., Hall S. R., Sanders E. P. \& Collier J. (1934) The wood feeding roach Cryptocerus, its protozoa, and the symbiosis between protozoa and roach. Mem. Am. Acad. Arts Sci. 17, 185-342.

Couturier S. (1961) Recherche anatomique et histologique sur l'iléon des Melolonthinae (Coleoptères Scarabaeides). Ann. Epiphyties 12, 317-346.

Cruden D. L. \& Markovetz A. 1. (1979) Carboxymethylcellulose decomposition by intestinal bacteria of cockronches. Appl ent. Microhiol. 38, 369-372.

Dixit A. \& MALL S. B. (1978) Digestive enzymes of mature larvae of Chilena similis Walker (Lepidoptera: Lasciocampidaej. Indian J. Ent. 39, 319-323.

Deschamps P. (1944) Sur la digestion du bois par les larves des cérambycides. Bull. Soc. ent. Fr. 49, 104-110.

ESENTHER G. R. \& KIRK T. K. (1974) Catabolism of aspen sapwood in Reticuliternes flavipes (Isoptera: Rhinotermitinae). Ann. ent. Soc. Am. 67, 989-990.

Evans W. A. L. \& PaYNI: D. W. (1964) Carbohydrases of the alimentary tract of the desert locust, Schistocerca aregaria. J. linsect Phisiol. 10, 657-674.
FALCK R. (1930) Die Scheindestruktion des Koniferenholzes durch die Larven des Hausbockes (Hylotrupes hajulus L.). Cellulose Chem. 11, 89-91.

Ferreira C. \& Terra W, R. (1980) Intracellular distribution of hydrolases in midgut caecae cells from an insect with emphasis on plasma membrane-bound enzymes. Comp. Biochem. Physiol. 66B, 467-473.

FISK F. W. \& RAO B. R. (1964) Digestive carbohydrases in the Cuban burrowing cockroach. Ant. ent. Soc. Am. 57, 40-44.

Ghose T., Montenecourt B. S. \& Eveleigil D. E. (1981) Measure of Cellulase Activity. International Union of Pure and Applied Chemistry, Commission of Biotechno$\log y$.

HonIGBERo B. M. (1970) Protozoa associated with termites and their role in digestion. In Biology of Termites (Edited by Krishna K. K. \& WeESNER F. M.), Vol. 2, pp. 1-36. Academic Press, New York.

HORI K. (1975) Digestive carbohydrases in the salivary gland and midgut of several phytophagous bugs. Comp. Biochem. Physial 50B, 145-151.

HUNGate R. E. (1938) Studies on the nutrition of Zootermopsis. II. The relative importance of the termite and the protozoa in wood digestion. Ecology 19, $1-25$.

Hungate R. E. (1943) Quantitative analyses on the cellulose fermentation by termite protozoa. Anr. ent. Soc. Am. 36, $730-739$.

IshaAya I. \& Plant H. N. (1974) Digestive enzymes in Eurytoma amyadali and their relation to food digestion and to the boring process of the emergence holes in almond fruits. Comp. Bioghen. Physiol. 48A, 3744.

IVANOVIC J. \& BARBIC F. (1966) Comparative studies of amylase and cellulase activity in insect larvae living in bark and wood. Arh. biol. Nauka 18. 1-2. Chem. Ahstr. 67, $1147 y(1967)$.

KHAN M. R. \& FORD J. B. (1967) The distribution and localization of digestive enzymes in the alimentary canal and salivary glands of the cotton stainer, Dysdercus fasciatus. J. Insect Physiol. 13, 1619-1628.

KhaN M. R. \& Kasting R. (1969) Digestive enzymes of sixth-instar larvae of the pale western cutworm, Ayrotis orthogonia. Can. Ent. 101, 494-499.

Kovoor J. (1970) Presence d'enzymes cellulolytique dans lintestin d'un termite supérieur Microcerotermes caltentatus (Was.). Amnls. Sci. Nat. (Zoologie) 12. 65-71.

Kukor J. J. \& MARTIN M. M. (1983) Siricid wood wasps acquire digestive enzymes from their fungal symbiont. Science, N.Y. (in press).

LASKeR R. \& GIESE A. C. (1956) Cellulose digestion by the silverfish Ctenolepisna lineata. J. exp. Biol 33, 542553 .

LEE K. E. \& WOODS T. G. (1971) Termites and Soils. Academic Press, New York.

Mall S. B., Singh A. R. \& Dixit A. (1978) Digestive enzymes of mature larvae of Atteva fabriciella (Lepidoptera: Yponomentidae) J. Anim. Morph. Physiol. 25, 86-92.

Mansour K. \& Mansour-Bek J. J. (1934a) On the digestion of wood by insects. J. exp. Biol. 11, 243-256.

Mansour K. \& Mansour-BeK J. J. (1934b) The digestion of wood by insects and the supposed role of microorganisms. Biol. Ret: 9, 363-382.

Mansour K. \& Mansour-BeK J. J. (1937) On the cellulase and other enzymes of Stromatium filmum Villers (Cerambycidae). Enzymologia 4, 1-6.

MARTIN M. M. (1984) The role of acquired enzymes in digestive processes in insects. In Animal-Microbial Interations (Edited by ANDERSON J. M., RAYNFR A. D. M. \& Walton D.), in press. Cambridge University Press, Cambridge.

Martin M. M. \& Martin J. S. (1978) Cellulose digestion in the midgut of the fungus-growing termite Macrotermes natalensis: the role of acquired digestive enzymes. Science. N.Y. 199, 1453-1455. 
MARTIN M. M. \& MARTIN J.S. (1979) The distribution and origins of the cellulolytic enzymes of the higher termite Macrotermes natalensis. Physiol. Zool. 52, 1-11.

Martin M. M., Kukor J. J., Martin J. S., Lawson D. L. \& Merritt R. W. (1981a) Digestive enzymes of larvae of three species of caddisflies (Trichoptera). Insect Biochem. 5, 50$\rfloor-505$

Martin M. M., Martin J. S., Kukor J. J. \& Merritt R. W. (1981b) The digestive enzymes of detritus-feeding stonefly nymphs (Plecoptera: Pteronarcyidae). Can. $J$. Zool. 59, 1947-1951.

Mathur L. M. L. (1966) Studies on the digestive enzymes of noctuid caterpillars feeding on cauliflower. Indian $J$. Ent. 28, 215-223.

MauldiN J. K. (1977) Cellulose catabolism and lipid synthesis in normally and abnormally faunated termites. Reticulitermes flavipes. Insect Biochem. 7, 27-31.

Mauldin J. K., Smythe R. V. \& Baxter C. C. (1972) Cellulose catabolism and lipid synthesis by the subterranean termite, Copiotermes formosamis. Insect Biochem. 2. 209-217.

McEwen S. E., Slaytor M. \& O’Brien R. W. (1980) Cellobiase activity in three species of Australian termites. Insect. Biochem. 10, 563-567.

MCGinnis A. J. \& Kasting R. (1969) Digestibility studies with cellulose-U-C $\mathrm{C}^{14}$ on larvae of the pale western cutworm, Ayrotis orthogonia. J. Insect Physiol. 15, 5-10.

Mishra S. C. (1979) Studies on deterioration of wood by insects: 4. Digestibility and digestion of major wood components by the termite Neotermes hosei (Isoptera Kalotermitide). Mater. Ory. 14, 269-278.

Mishra S. C. (1980) Carbohydrases in Neotermes bosei Snyder (Isoptera: Kalotermitidae). Mater. Org. 15, $253-261$

Mishra S. C. \& Singh P. (1978) Polysaccharide digestive enzymes in the larvae of Stronatium barbatum (Fabr.) a dry wood borer (Coleoptera: Cerambycidae). Mater. Org. 13, 115-122.

Misra J. N. \& Ranganathan V. (1954) Digestion of cellulose by the mound building termite, Termes $(\mathrm{Cg}$ lotermes) obesus (Rambur). Proc. Indian Acad. Sci. 39B, $100-113$.

MONK D. C. (1976) The distribution of cellulase in freshwater invertebrates of different feeding habits. Freshwater Biol. 6, $471-475$.

MORGAN M. R. J. (1975a) A qualitative survey of the carbohydrases of the alimentary tract of the migratory locust, Locusta migratoria migratorioides. J. Insect. Physiol. 21, 1045-1053

MORGAN M. R. J. (1975b) Relationship between gut cellobiase, lactase, aryl $\beta$-glucosidase and ayrl $\beta$-galactosidase activities of Locusta migratoria. Insect. Biochem. 5 , 609-619.

MoRGaN M. R. J. (1976) Gut carbohydrases in locusts and grasshoppers. Acrida 5, 45-58.

Mukalyama F. (1961) Occurrence of several digestive enzymes in the salivary gland of the larva of the silkworm, Bombyx mori. Nippon Sanshigaku Zashi 30, 1-8. Chem. Ahstr. 59. 11940d (1963).

MüLleR W. (1934) Untersuchungen über die Symbiose von Tieren mit Pilzen und Bakterien. Archs. Mikrohiol. 5. 84-147.

NifLSEN C. O. (1962) Carbohydrases in soil and litter invertebrates. Oikos 13, 200-215.

Norman A. G. (1936) The destruction of oak by the death watch beetle. Biochem. J. 30, 1135-1137.

O'Brien R. W. \& Slaytor M. (1982) Role of microorganisms in the metabolism of termites. Aust. J. biol. Sci. 35, 239-262.

O'Brien G. W., Veivers P. C., McEwen S. E., Slaytor M \& O'BRIEN R. W. (1979) The origin and distribution of cellulase in the termites, Nasutitermes exitiosus and Coptotermes lacteus. Insect. Biochem. 9, 619-625.
Orlova E. A. (1974) Influence of the intestinal symbiont complex on the intensity of food consumption and the longevity of the termites Reticulitermes. In Termites (Collected Articles). (Edited by ZoLotarev E. K.). Transactions of the Entomological Division No. 5, pp. 165-180. University Publishing House, Moscow.

PARKIN E. A. (1940) The digestive enzymes of some woodboring beetle larvae. $J$, exp. Biol. 17, 364-377.

Potts R. C. \& HewitT P. H. (1973) The distribution of intestinal bacteria and cellulase activity in the harvester termite Trinervitermes trinervoides (Nasutitermitinae). Insectes soc. 20, 215-220.

Potts R. C. \& Hewitt P. H. (1974a) The partial purification and some properties of the cellulase from the termite Trinervitermes trinervoides (Nasutitermitiae). Comp Biochem. Physiol. 47B, 317-326.

Potts R. C. \& HewitT P. H. (1974b) Some properties and reaction characteristics of the partially purified cellulase from the termite Trinervitermes trinervoides (Nasutitermitinae). Comp. Biochem. Physiol. 47B, 327-337.

REESE E. T. \& MANDELS M. (1971) Degradation of cellulose and its derivatives; enzymic degradation. In High Polymers (Edited by Bikales N. M. \& Segal L.) 2nd edn, Vol. 5. pp. 1079-1094. Wiley-Interscience, New York.

Reyes V. G. \& Tiedje J. M. (1976a) Ecology of the gut microbiota of Tracheoniscus rathkei (Crustacea, Isopoda). Pedobiologia 16, 6774 .

REyes V. G. \& TIEDJE J. M. (1976b) Metabolism of ${ }^{14} \mathrm{C}$-labelled plant materials by woodlice (Tracheoniscus rathke Brandt) and soil microorganisms. Soil Biol. Biochem. 8, 103-108.

Ripper W. (1930) Zur Frage des Celluloseabbaus der Holzverdauung xylophager Insektenlarven. $Z$. vergl. Physiol. 13, 314-333

Rivnay M. S. (1945) Physiological and ecological studies of the species of Capnodis in Palestine (Col., Buprestidae). II - studies on the larvae. Bull. ent. Res. 36, 103-119.

RösSLER M. E. (1961) Ernahrungsphysiologische Untersuchungen an Scarabaeidlarven (Oryctes nasicornis L. Melolonthu melolontha L.). J. Insect. Physinl 6, 62-80.

Srhlottke E. (1945) Uber die Verdauungsfermente im Holzfressender Käferlarven, Zool. Jb., Allgemeine Zoologie und Physiologie der Tiere 61, 88-140.

Schulze E. F. \& ERhardt P. (1963) Nachweis Kohlenhydratspaltender Fermente im Darmtrakt verschiedener Entwicklungsstadian von Diprion pini L. (Hym., Tenthredinidae). Ent. exp. appl. 6, 114-122.

Seifert K. \& Becker G. (1965) Der chemische Abbau von Laub- und Nadelholzarten durch verschiedenden Termiten. Holsforsehung 19, 105-111.

SunvodA $O$. (1930) Contributions to the knowledge of intestinal secretion in insects-III. On the digestive enzymes of the silkworm. J. Biochem., Tokyo 11, 345-367.

Sinha T. B. \& SRivastava D. C. (1970) Cellulose digestion in Pserudocacilius elutus (Psocoptera). Enzymologia 39, $84-86$.

Sinsabaugh R. L., Benfield E. F. \& Linkins A. E. (1981) Cellulose digestion and assimilation by a stream shredder. Abstract, 29th Anmul Meeting of the North American Benthological Society, Provo, Utah, April $27-30,1981$

Soo Hoo C. F. \& Dudzinski A. (1967) Digestion by the larvae of the pruinose searab. Ent. exp. Appl. 10, 7-15.

SPILLFR D. (1951) Digestion of alpha-cellulose by larvae of Anobium munctalum DeGeer. Nature, Lond. 168, 209-210.

TAKANONA T. \& HORI K. (1974) Digestive enzymes in the salivary gland and midgut of the bug Stenotus binotatus. Comp. Biochem. Physiol. 47A, 521-528.

Talmadge K. W. \& Albersheim P. (1969) Plant cell wall polysaccharide degrading enzymes of Melanoplus bivittatus. J. Insect Physiol. 15, 2273-2283.

Terra W, R., Ferreira C. \& Debianchi A. G. (1979) Dis- 
tribution of digestive enzymes among the endo- and ectoperitrophic spaces and midgut cells of Rhynchosciara and its physiological significance. J. Insect Physiol. 25, 487-494.

ThAYtR D. W. (1978) Carboxymethylcellulase produced by facultative bacteria from the hindgut of the termite Reticulitermes hesperus. J. gen. Microbiol. 106, 13-18.

Trager W. (1932) A cellulase from the symbiotic intestinal flagellates of termites and of the roach. Cryptocercus punctulatus. Biochem. J. 26, 1762-1771.

Vitigers P. C.. Musca A. M., O’Brien R. W. \& Slaytor M. (1981) Digestive enzymes of the salivary glands and gut of Mastotemes darwiniensis. Insect Biochem. 12, $35-40$.

Wharton D. R. A. \& Wharton M. L. (1965) 'The cellulase content of various species of cockroaches. J. Insect Phvsiol. 11, 1401-1405.

Wiejomanin J. Г. (1930) Die Celluloseverdauung bei Lamellicornierlarven. Z. Morph. Okol. Tiere 19, 228-258.

W(x)I T. M. \& MCCRAE S. I. (1979) Synergism between enzymes involved in the solubilization of native cellulose. In Hydrolysis of Cellulose: Mechanisms of Enzymatic and Acid Hydrolysis (Edited by BRown R. D. \& JURASEK L.). Advances in Chemistry Series, No. 181, pp. 179-209. American Chemical Society, Washington.

Yamaoka I. \& Nagatani Y. (1975) Cellulose digestion system in the termite Reticulitermes speratus (Kolbe). I. Producing sites and physiological significance of two kinds of cellulase in the worker. Zool. Mag., Tok yo 84, 23-29.

YAMIN M. A. (1981) Cellulose metabolism by the flagellate Trichonympha from a termite is independent of endosymbiotic bacteria. Science, N.Y. 211, 58-89.

YAMIN M. A. \& TraGer W. (1979) Cellulolytic activity of an axenically-cultivated termite flagellate, Trichomitopsis termopsidis. J. gen. Microbiol. 13, 417-420.

YOKOE Y. (1964) Cellulase activity in the termite Leucotermes speratus with new evidence in support of a cellulase produced by the termite itsell. Scientific Papers of the College of General Education, Unversity of Tokyo 14. $115-120$ 